\title{
Multi-parametric MRI zone-specific diagnostic model performance compared with experienced radiologists for detection of prostate cancer
}

\author{
Nikolaos Dikaios ${ }^{1,2} \cdot$ Francesco Giganti $^{3,4} \cdot$ Harbir S. Sidhu ${ }^{1} \cdot$ Edward W. Johnston ${ }^{1} \cdot$ Mrishta B. Appayya ${ }^{1} \cdot$ \\ Lucy Simmons $^{5} \cdot$ Alex Freeman $^{6} \cdot$ Hashim U. Ahmed $^{7} \cdot$ David Atkinson $^{1,3} \cdot$ Shonit Punwani $^{1,3}$ (D)
}

Received: 29 May 2018 / Revised: 4 September 2018 / Accepted: 24 September 2018 / Published online: 19 November 2018

(C) The Author(s) 2018

\begin{abstract}
Objectives Compare the performance of zone-specific multi-parametric-MRI (mp-MRI) diagnostic models in prostate cancer detection with experienced radiologists.

Methods A single-centre, IRB approved, prospective STARD compliant 3 T MRI test dataset of 203 patients was generated to test validity and generalisability of previously reported $1.5 \mathrm{~T}$ mp-MRI diagnostic models. All patients included within the test dataset underwent $3 \mathrm{~T}$ mp-MRI, comprising T2, diffusion-weighted and dynamic contrast-enhanced imaging followed by transperineal template \pm targeted index lesion biopsy. Separate diagnostic models (transition zone (TZ) and peripheral zone (PZ)) were applied to respective zones. Sensitivity/specificity and the area under the receiver operating characteristic curve (ROC-AUC) were calculated for the two zone-specific models. Two radiologists (A and B) independently Likert scored test $3 \mathrm{~T}$ mp-MRI dataset, allowing ROC analysis for each radiologist for each prostate zone.

Results Diagnostic models applied to the test dataset demonstrated a ROC-AUC $=0.74$ (95\% CI 0.67-0.81) in the PZ and 0.68 (95\% CI 0.61-0.75) in the TZ. Radiologist A/B had a ROC-AUC $=0.78 / 0.74$ in the PZ and 0.69/0.69 in the TZ. Radiologists A and $\mathrm{B}$ each scored 51 patients in the $\mathrm{PZ}$ and 41 and 45 patients respectively in the $\mathrm{TZ}$ as Likert 3 . The $\mathrm{PZ}$ model demonstrated a ROC-AUC $=0.65 / 0.67$ for the patients Likert scored as indeterminate by radiologist $\mathrm{A} / \mathrm{B}$ respectively, whereas the $\mathrm{TZ}$ model demonstrated a ROC-AUC $=0.74 / 0.69$.

Conclusion Zone-specific mp-MRI diagnostic models demonstrate generalisability between 1.5 and $3 \mathrm{~T}$ mp-MRI protocols and show similar classification performance to experienced radiologists for prostate cancer detection. Results also indicate the ability of diagnostic models to classify cases with an indeterminate radiologist score.

Key Points

- MRI diagnostic models had similar performance to experienced radiologists for classification of prostate cancer.

- MRI diagnostic models may help radiologists classify tumour in patients with indeterminate Likert 3 scores.
\end{abstract}

Keywords Magnetic resonance imaging $\cdot$ Prostatic neoplasms $\cdot$ Diagnosis $\cdot$ Computer-assisted $\cdot$ Logistic models

\section{Abbreviations}

AUC Area under the curve

CAD Computer-assisted diagnosis

Shonit Punwani

shonit.punwani@gmail.com

1 Centre for Medical Imaging, University College London, 2nd floor, Charles Bell House, 43-45 Foley Street, London W1W 7TS, UK

2 Centre for Vision, Speech and Signal Processing, University of Surrey, 388 Stag Hill, Guildford GU2 7XH, UK

3 Departments of Radiology, University College London Hospital, 235 Euston Road, London NW1 2BU, UK
DCE Dynamic contrast-enhanced

DCE-nSI Early contrast-enhanced T1 signal intensity

DWI Diffusion-weighted imaging

4 Division of Surgery \& Interventional Science, University College London, London, UK

5 Research Department of Urology, Division of Surgery and Interventional Science, University College London, London NW1 2PG, UK

6 Department of Histopathology, University College London Hospital, London NW1 2PG, UK

7 Department of Surgery and Cancer, Imperial College London, London, UK 


$\begin{array}{ll}\text { LR } & \text { Logistic regression } \\ \text { ME } & \text { Maximum enhancement } \\ \text { mp } & \text { Multi-parametric } \\ \text { PSA } & \text { Prostate-specific antigen } \\ \text { PZ } & \text { Peripheral zone } \\ \text { ROC } & \text { Receiver operator characteristic } \\ \text { ROI } & \text { Region of interest } \\ \text { SI } & \text { Signal intensity } \\ \text { T2-nSI } & \text { Normalised T2 signal intensity } \\ \text { TPM } & \text { Template mapping biopsy } \\ \text { TRUS } & \text { Transrectal ultrasound-guided biopsy } \\ \text { TZ } & \text { Transition zone }\end{array}$

\section{Introduction}

Multi-parametric MRI (mp-MRI) has heralded a paradigm shift in the management of prostate cancer. It is now commonly employed to localise suspicious areas within the prostate and facilitate targeted histological sampling [1]. Nevertheless, mpMRI remains an imperfect test. For example, between 30 and $40 \%$ of mp-MRI studies, even by experienced radiologists, are scored as indeterminate for cancer (Likert/PIRADS 3) [2,3]. An indeterminate mp-MRI confers little benefit. Most patients with indeterminate mp-MRI-scored studies do not have cancer within the gland [3]. However, a significant minority harbour small volume Gleason $3+4$ disease or more widespread Gleason $3+3$ disease [4]. An indeterminate study results in a management dilemma of whether to perform a biopsy. Not performing the biopsy risks underdiagnosing patients with significant prostate cancer, whilst performing a biopsy risks over the investigation of patients with likely insignificant or no cancer. Furthermore, mp-MRI also misses approximately $10 \%$ of cases of significant prostate tumour [4]. There remains a need to improve the performance of mp-MRI.

One potential approach to address these challenges has been to develop diagnostic models based on quantitative mp-MRI metrics. For example, we have previously derived zone-specific logistic regression (LR) models for classification of significant prostate cancer $[5,6]$. Others have also developed similar models [7-9]. However, whilst studies based on internal validations suggest an overall good performance, it is recognised that this performance may be an overestimate and not generalisable to other (external) datasets.

Within this study, we aimed to assess the external validity of our previously derived and internally validated mp-MRI LR models $[5,6]$. Specifically, we apply the zone-specific LR models, derived on a Siemens 1.5 T mp-MRI dataset, for the classification of an independent cohort of patients imaged using a Philips 3 T scanner.

In order to evaluate the potential clinical value of LR models, we compare their overall performance against experienced radiologists for classification of patients with significant prostate cancer and determine whether LR models could be applied for the classification of indeterminate (Likert $3 / 5$ ) scored cases.

\section{Material and methods}

Our local institutional review board approved the study and waived the requirement for individual consent for retrospective analysis of patient data collected as part of clinical trials/ routine care (R\&D No: 12/0195; date: 16 July 2012).

\section{Patient population}

A single centre, IRB approved, prospective STARD compliant trial dataset of 330 patients [4] was reviewed. Three hundred thirty men (median age of 63 years, interquartile range, IQR [42-83]; median prostate-specific antigen (PSA) of $7.4 \mathrm{ng} / \mathrm{ml}$, IQR [0.7-58.05]), with previous negative/ nonsignificant prostate disease on transrectal ultrasound (TRUS) biopsies, but in whom a clinical suspicion of prostate cancer remained, were consecutively enrolled from 11 January 2012 to 29 January 2014. All patients underwent $3 \mathrm{~T}$ mp-MRI (Achieva, Philips Healthcare) of the prostate. All studies were prospectively scored using a Likert scale by an experienced radiologist (radiologist A; with 14 years of experience in prostate mp-MRI and reporting more than $500 \mathrm{mp}$-MRI scans/ year). Patients then underwent transperineal template mapping biopsies of the whole gland (irrespective or radiologist report) \pm MR-targeted biopsy of a suspected index lesion (based on the radiologist report) as described within the PICTURE study protocol [4]. In summary, mapping using $5 \mathrm{~mm}$ sampling was obtained using core needles inserted via a brachytherapy grid fixed on a stepper. In most prostates, two biopsies at each grid point were required to sample the full craniocaudal gland length. Two to three targeted biopsies were performed for the mp-MRI index lesion-scored Likert 3 or above. In total, $90 \%$ of the patients underwent the additional targeted biopsy.

To derive a complete dataset of patients scanned at $3 \mathrm{~T}$, men were selected with the following criteria: (i) a full template biopsy \pm targeted biopsy and (ii) a complete $3 \mathrm{~T}$ mp-MRI comprising of $\mathrm{T} 2 \mathrm{~W}$, diffusion-weighted (DW) and dynamic contrast-enhanced (DCE) imaging. One hundred twentyseven patients did not meet the inclusion criteria; 5 had mpMRI performed at $1.5 \mathrm{~T}, 11$ had incomplete mp-MRI datasets, 71 did not undergo the full template biopsy due to clinical circumstances, 13 patients were excluded due to post-biopsy artefacts, 12 patients were excluded due to distorted DWI and 15 patients were excluded where cancer was present in both the PZ and the TZ (Fig. 1). Two hundred three patients with median age 65.4 years (interquartile range, $\mathrm{IQR}=42.9-86$ ) and median PSA of $7.2 \mathrm{ng} / \mathrm{ml}(\mathrm{IQR}=2.1-32.4)$ formed the final test dataset. 
Train mpMRI LR model to predict prostate Cancer

Interrogation of patient archive imaged at 1.5T MRI

\section{6 selected $\mathrm{PZ}$ patients (median PSA of $6.5 \mathrm{ng} / \mathrm{ml}$, IQR [4.8-10]) with:}

(1) $\mathrm{mpMRI}$ of the prostate

(2) matched TPM biopsies of the gland

(3) biopsy confirmed PZ cancer ( $n=20$ 'significant $\mathrm{Ca}^{\prime}, \mathrm{n}=56$ 'benign')

\section{PZ Training Cohort}

Train mpMRI LR model to detect $P Z$ cancer

\section{Validate mpMRI LR models}

Interrogation of independent patient archive imaged at 3T MRI. Patients with complete $\mathrm{mpMRI}$ and full template biopsy \pm targeted biopsy were included.

203 selected patients (median PSA of $7.2 \mathrm{ng} / \mathrm{ml}$, IQR [2.132.4]) with:

(1) $\mathrm{mpMRI}$ of the prostate

(2) matched TPM biopsies of the gland

(3) biopsy confirmed cancer ( 89 had 'significant Ca' within the TZ and 110 within the PZ)
Validate $\mathrm{mpMRI} L \mathrm{R}$ model on $\mathrm{PZ}$ cancer detection subanalysis on $51 / 51$ cases where radiologists $A / B$ were uncertain (Likerts3/5)
Validate mpMRI LR model on TZ cancer detection subanalysis on $41 / 45$ cases where radiologists $A / B$ were uncertain (Likerts3/5)
Fig. 1 Flowchart outlining the study

Of the 203 men included in the final dataset, 89 had biopsyconfirmed significant cancer (see the "Histological reference standard" section) within the TZ and 110 biopsies confirmed significant cancer within the PZ. Median PSA of patients with significant cancer within the PZ and TZ was $7.22 \mathrm{ng} / \mathrm{ml}$ and $6.52 \mathrm{ng} / \mathrm{ml}$ respectively. Median PSA of patients with benign histology or non-significant cancer only within the PZ and TZ was $7.02 \mathrm{ng} / \mathrm{ml}$ and $7.77 \mathrm{ng} / \mathrm{ml}$ respectively.

A second radiologist (radiologist $\mathrm{B}$, with 5 years of experience in prostate mp-MRI and reporting more than $1200 \mathrm{mp}$ MRI scans/year) retrospectively re-reported each of the 203 patient's study, unaware of radiologist A's original report and histological results.

As Likert scoring has been prospectively validated within multicentre PROMIS trial [10] and is the scoring method recommended by UK consensus [11], both radiologists (A and B) used the Likert scale to score the presence of significant disease within each prostate zone.

\section{Multi-parametric MRI protocol}

The full details of the mp-MRI performed as part of the PICTURE trial have been previously reported [4]. In brief, MRI was performed on a single $3 \mathrm{~T}$ scanner (Achieva, Philips Healthcare) using a 32-channel cardiac phased-array coil. Prior to imaging, $0.2 \mathrm{mg} / \mathrm{kg}$ (up to $20 \mathrm{mg}$ ) of a spasmolytic agent (Buscopan; Boehringer Ingelheim) was administered intravenously to reduce bowel peristalsis. Axial and coronal T2-weighted images were acquired with $\mathrm{TR} / \mathrm{TE}=5407 /$ $100 \mathrm{~ms}$, flip angle $=90^{\circ}$, field of view $=180 \mathrm{~mm}$, a $3-\mathrm{mm}$ slice thickness and slice centre-to-centre separation of $3 \mathrm{~mm}$. Axial DW images were acquired at $b=0,150,500,1000$, and $2000 \mathrm{~s} / \mathrm{mm}^{2}$ with TR $/ \mathrm{TE}=2753 / 80 \mathrm{~ms}$, flip angle $=90^{\circ}$, field of view $=220 \mathrm{~mm}$ and a $5-\mathrm{mm}$ slice thickness. A high $b$ value at $b=2000 \mathrm{~s} / \mathrm{mm}^{2}$ was included to evaluate the interstitial free water and permeability. An axial apparent diffusion coefficient (ADC) map was generated automatically from DW images at $b=0,150,500$, and $1000 \mathrm{~s} / \mathrm{mm}^{2}$. DCE was performed with a T1-weighted volumetric sequence $(\mathrm{TR} / \mathrm{TE}=5.8 / 28 \mathrm{~ms}$, flip angle $=10^{\circ}$, field of view $=180 \mathrm{~mm}$, slice thickness $=$ $3 \mathrm{~mm}$, temporal resolution of $15 \mathrm{~s}$ ) before and after intravenous administration of at least $0.1 \mathrm{mmol} / \mathrm{kg}$ gadolinium meglumine contrast agent (Dotarem $®$, Guerbet) at a rate of $3 \mathrm{ml} / \mathrm{s}$ via power injector, followed by $20 \mathrm{ml}$ saline bolus at the same rate.

\section{Radiologist Likert scoring}

Radiologists used a 12-segment (anterior/posterior, left/right, and division into the apical/middle/basal thirds) prostate pictorial reporting proforma to provide Likert scores for each segment together with drawing and scoring any identified focal lesions. For each patient, the highest Likert score given by each radiologist for each zone is summarised in Table 1. Figures 2 and 3 (in the PZ and the TZ respectively) show examples of the regions with positive template mapping biopsy (TPM) biopsy that were independently scored as indeterminate $($ Likert $=3$ ) by both experienced radiologists.

\section{Histological reference standard}

The PICTURE study employed TPM biopsy \pm targeted biopsy of the suspected index lesion as previously described [4]. A TPM histological reference standard (as compared with prostatectomy reference) provides the opportunity to collect truenegative as well as true-positive cases. It has a reported sensitivity of $95 \%$ and negative predictive value of $95 \%$ for clinically significant cancers of volume $>0.5 \mathrm{~cm}^{3}$ and $76 \%$ sensitivity for all cancers [12-14].

An experienced (12 years) prostate pathologist analysed all biopsy cores blinded to the mp-MRI findings. Significant cancer was defined, based on previously reported TPM biopsy 
Table 1 Radiologists A and B Likert scoring for the presence of significant cancer within the $\mathrm{PZ}$ and $\mathrm{TZ}$ in the cohort of patients scanned at $3 \mathrm{~T}$

\begin{tabular}{|c|c|c|c|c|c|c|c|}
\hline & \multicolumn{7}{|c|}{ Distribution of PZ Likert scores } \\
\hline & & \multicolumn{6}{|c|}{ Radiologist B Likert score } \\
\hline & & 1 & 2 & 3 & 4 & 5 & Total \\
\hline \multirow[t]{5}{*}{ Radiologist A Likert score } & 1 & 0 & 1 & 0 & 0 & 1 & 2 \\
\hline & 2 & 0 & 31 & 9 & 0 & 5 & 45 \\
\hline & 3 & 0 & 6 & 37 & 4 & 4 & 51 \\
\hline & 4 & 0 & 3 & 3 & 31 & 12 & 49 \\
\hline & 5 & 0 & 1 & 2 & 6 & 47 & 56 \\
\hline \multirow[t]{4}{*}{ Total } & & 0 & 42 & 51 & 41 & 69 & 203 \\
\hline & \multicolumn{7}{|c|}{ Distribution of TZ Likert Scores } \\
\hline & & \multicolumn{6}{|c|}{ Radiologist B Likert score } \\
\hline & & 1 & 2 & 3 & 4 & 5 & Total \\
\hline \multirow[t]{5}{*}{ Radiologist A Likert score } & 1 & 0 & 0 & 0 & 2 & 0 & 2 \\
\hline & 2 & 0 & 97 & 18 & 4 & 2 & 121 \\
\hline & 3 & 0 & 11 & 26 & 3 & 1 & 41 \\
\hline & 4 & 0 & 0 & 0 & 6 & 3 & 9 \\
\hline & 5 & 0 & 2 & 1 & 1 & 26 & 30 \\
\hline Total & & 0 & 110 & 45 & 16 & 32 & 203 \\
\hline
\end{tabular}

criteria [15], as the presence of any Gleason 4 tumour (primary or secondary pattern) or Gleason $3+3$ tumour with a maximum cancer core length threshold $4 \mathrm{~mm}$.

\section{Quantitative evaluation of $\mathrm{mp}-\mathrm{MRI}$}

The test mp-MRI datasets were analysed with MIM Symphony Version 6.1 (MIM Software Inc.). Following radiologist scoring, the two radiologists were unblinded to each other's mp-MRI reports and the histological reference standard. The radiologists, in consensus, manually contoured the regions of interest (ROI) on mp-MRI (T2-weighted images, apparent diffusion coefficient (ADC) maps and contrast earlyenhanced T1-weighted images) to allow the extraction of quantitative mp-MRI metrics from regions of significant cancer and also from areas confirmed to have benign histology.

Specifically, where visible, the index lesion containing significant cancer within each zone (PZ and $\mathrm{TZ}$ ) was contoured on each imaging sequence. Where no lesion was evident (even in retrospect) on mp-MRI and histological confirmed significant cancer was present, the radiologists contoured a $1-\mathrm{cm}^{2}$ ROI at the histologically confirmed location of cancer. Where no lesion was visualised and/or no significant cancer present within a zone/lesion, the radiologists avoided the areas with histologically confirmed insignificant disease and contoured a $1-\mathrm{cm}^{2}$ ROI at a histologically confirmed benign location.

The mean signal intensity (SI) of each ROI on the corresponding T2-weighted images, ADC maps and early T1 arterial contrast-enhanced images was recorded. $\mathrm{T} 2$ and early $\mathrm{T} 1$ arterial contrast-enhanced SI were normalised against a right obturator internus ROI to give normalised T2 (T2-nSI) and normalised early dynamic contrast-enhanced SI (DCE-nSI) parameters. The maximum enhancement (ME) parameter was also derived from the DCE signal enhancement time curve as previously reported [16].

\section{Application of LR diagnostic models}

Previously derived $[5,6]$ zone-specific LR models (as below) were applied to calculate a predictive probability from the quantitative mp-MRI metrics extracted at each ROI anatomical location (ADC, T2nSI, ME for the TZ and, ADC, T2nSI and DCEnSI for the PZ).

$\mathrm{TZ}$ model $: \ln ($ Odds $)=5.347+0.332 \cdot \mathrm{ADC}-0.974 \cdot \mathrm{T} 2 \mathrm{nSI}-1.730 \cdot \mathrm{ME}$

PZ model $: \ln ($ Odds $)=-2.441-0.968 \cdot \mathrm{ADC}-0.200 \cdot \mathrm{T} 2 \mathrm{nSI}$

$$
+2.546 \cdot \mathrm{DCEnSI}
$$

Two separate probability thresholds were then used to classify an ROI anatomical location as positive or negative by the LR model.

The first threshold was based on the Youden index $(J)$ applied to the published model derivation $1.5 \mathrm{~T}$ mp-MRI datasets [5,6], where $J$ is a function of sensitivity and specificity and is defined as the maximum vertical distance between the ROC curve and the diagonal line [17-20]. $J$ occurs at the threshold probability for optimal diagnostic models classification ability. The maximum $J$ within the $\mathrm{PZ}$ was $J=$ 0.50 , related to a probability threshold $=17 \%$. For the $\mathrm{TZ}$ maximum, $J$ was 0.56 , with probability threshold $=31 \%$. 
Fig. 2 Axial multi-parametric MR images (a T2-weighted, b apparent diffusion coefficient map, c pre-contrast $\mathrm{T} 1$ and $\mathbf{d}$ early post-contrast $\mathrm{T} 1$ ) of a region in the peripheral zone scored as equivocal (diffused lesion; positive tumour TPM biopsy) by the radiologists
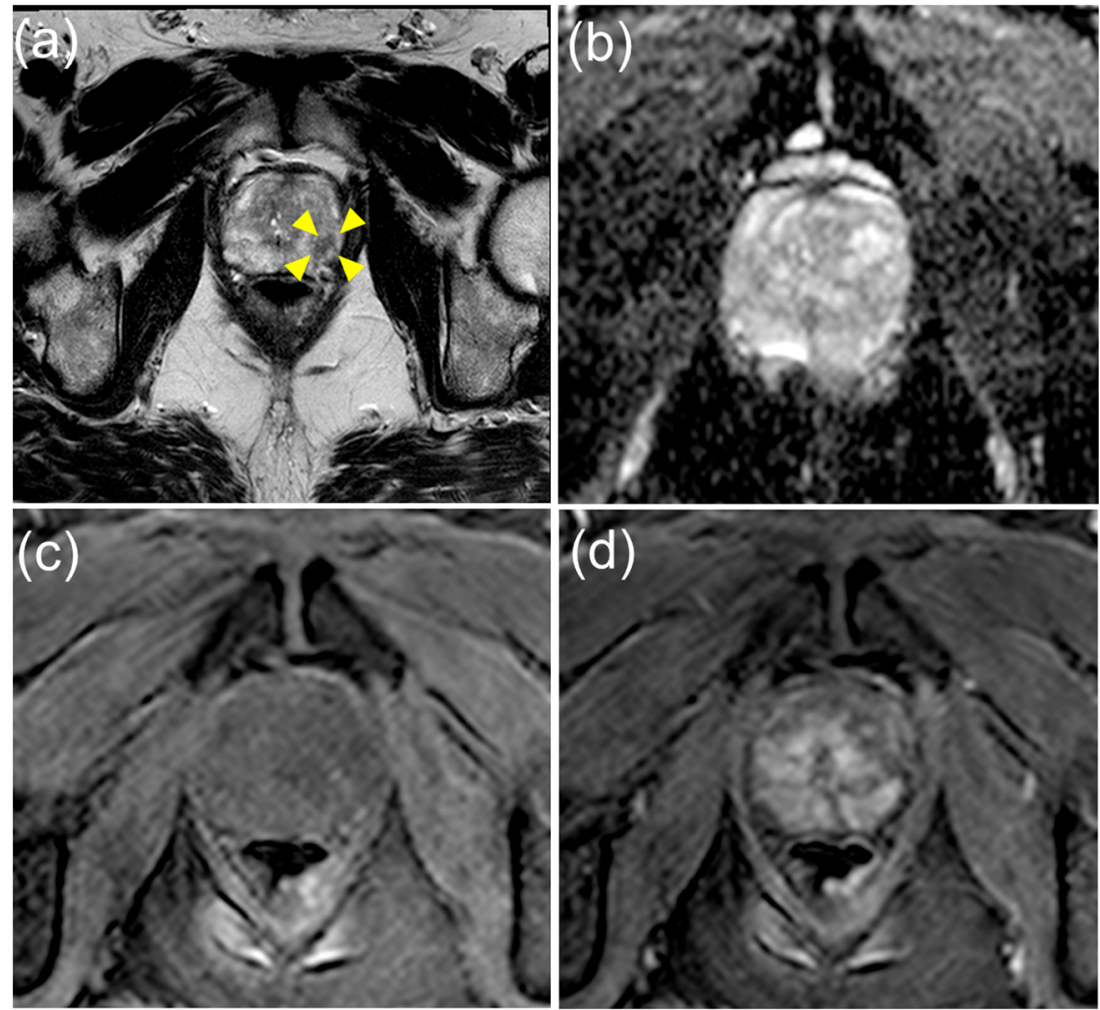

The second threshold was set at a specificity of 50\% from the ROC analysis performed previously on at the time of model derivation using the original $1.5 \mathrm{~T}$ mp-MRI dataset $[5,6]$. At this specificity, the estimated probability threshold for the significant disease was $14 \%$ for the $\mathrm{PZ}$ and $23 \%$ for the $\mathrm{TZ}$.

\section{Results}

The ROC-AUC of the PZ and TZ LR models when applied to the test dataset mp-MRI ROI anatomical location quantitative metrics was $0.74 \pm 0.04(\mathrm{SD})$ and $0.68+0.04(\mathrm{SD})$
Fig. 3 Axial multi-parametric MR images (a T2-weighted, b apparent diffusion coefficient map, c pre-contrast $\mathrm{T} 1$ and $\mathbf{d}$ early post-contrast $\mathrm{T} 1$ ) of a region (yellow arrows) in the transition zone scored as equivocal (positive tumour TPM biopsy) by the radiologists
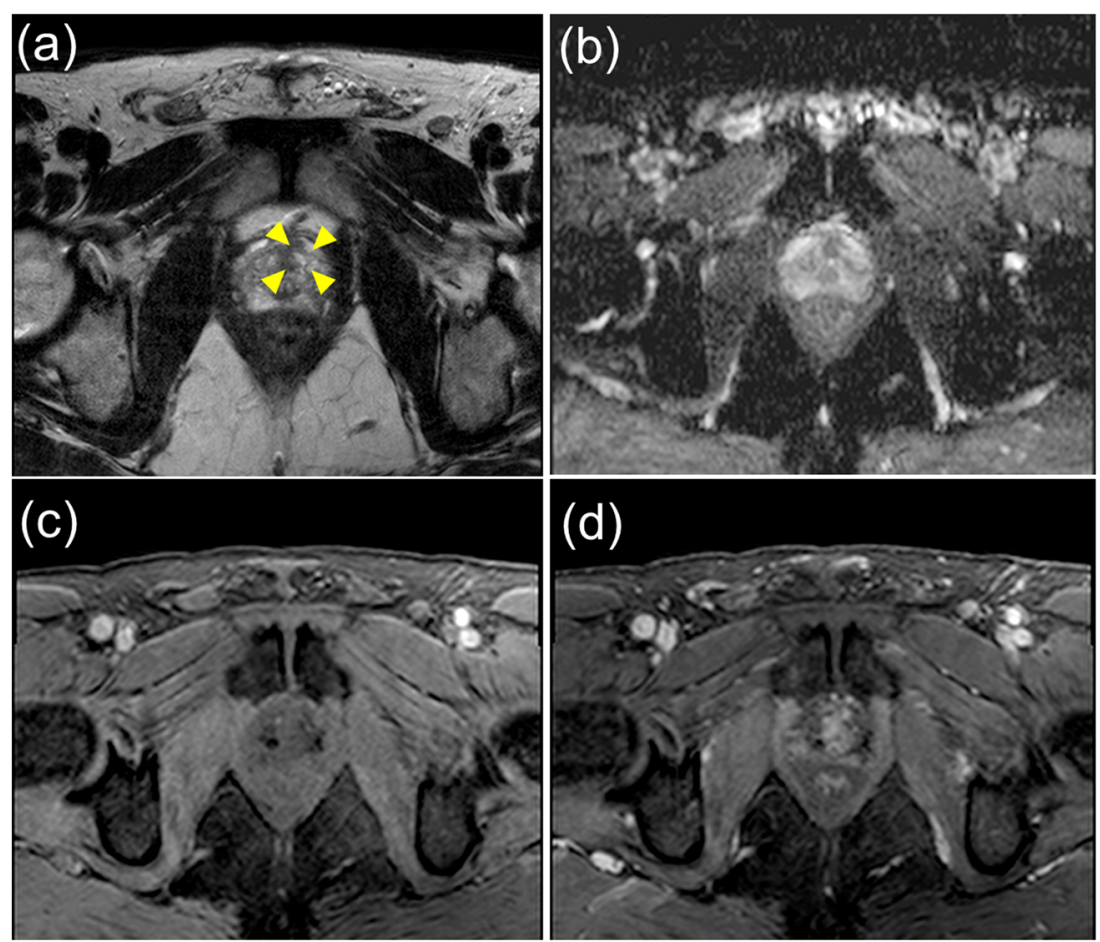
respectively. The ROC-AUC for the zone-specific LR models was similar to that for the experienced radiologist's Likert scores (Table 2). The agreement between radiologists A and B Likert scores, calculated using Cohen's kappa, was good both in the PZ $(\kappa=0.625)$ and the TZ $(\kappa=0.609)$.

The sensitivities/specificities of the PZ model using the Youden index and $50 \%$ specificity probability thresholds were $0.84 / 0.57$ and $0.90 / 0.51$ respectively, and for the TZ model were $0.84 / 0.32$ and $0.90 / 0.23$ respectively (Table 3 ). Radiologists A and B had sensitivities/specificities of $0.91 /$ 0.40 and $0.90 / 0.33$ in the PZ respectively and $0.58 / 0.75$ and $0.60 / 0.65$ in the TZ respectively (Likert $\geq 3$ scored as positive)

The PZ LR model with Youden index threshold classified $9 \%$ of patients as false positive and $20 \%$ as false negative; and with $50 \%$ specificity probability threshold, $5 \%$ as false positive and $23 \%$ as false negative. In comparison, using a Likert score threshold of $\geq 3$ within the $\mathrm{PZ}$ as positive, radiologist $\mathrm{A}$ classified $5 \%$ of patients as false positive and $28 \%$ as false negative, whilst radiologist B classified $5 \%$ as false positive and $31 \%$ as false negative.

The TZ LR model with Youden index threshold classified $7 \%$ of patients as false positive and $38 \%$ as false negative and with $50 \%$ specificity probability threshold, $4 \%$ as false positive and $43 \%$ as false negative. In comparison, using a Likert score threshold of $\geq 3$ within the $\mathrm{TZ}$ as positive, radiologist $\mathrm{A}$ classified $18 \%$ of patients as false positive and $14 \%$ as false negative, whilst radiologist B classified $18 \%$ as false positive and $20 \%$ as false negative.

Radiologist A scored $51 \mathrm{PZ}$ cases and $41 \mathrm{TZ}$ cases as indeterminate (Likert 3/5). Application of LR models to the Likert 3/5 group yielded a ROC-AUC 0.65 (95\% CI 0.50 0.80 ) for the PZ and ROC-AUC 0.74 (95\% CI $0.58-0.90$ ) for the TZ (Figs. 4 and 5). The model correctly classified $86 \%$ of $\mathrm{PZ}$ cases and $64 \%$ of $\mathrm{TZ}$ cases.

Radiologist B scored $51 \mathrm{PZ}$ cases and $45 \mathrm{TZ}$ cases as indeterminate (Likert 3/5). Application of LR models to the Likert 3/5 group yielded a ROC-AUC 0.67 (95\% CI 0.51-

Table 2 ROC AUC of PZ and TZ LR models and comparative experienced radiologist performance for classification of significant cancer

\begin{tabular}{lllll}
\hline & & & Lower bound & Upper bound \\
\hline $\begin{array}{l}\text { Peripheral zone } \\
\text { PZ model }\end{array}$ & 0.74 & 0.04 & 0.67 & 0.81 \\
Radiologist A & 0.78 & 0.03 & 0.72 & 0.84 \\
Radiologist B & 0.74 & 0.04 & 0.67 & 0.81 \\
Transition zone & & & & \\
TZ model & 0.68 & 0.04 & 0.61 & 0.75 \\
Radiologist A & 0.69 & 0.04 & 0.61 & 0.77 \\
Radiologist B & 0.69 & 0.04 & 0.62 & 0.77 \\
\hline
\end{tabular}

0.82) for the PZ and a ROC-AUC 0.69 (95\% CI 0.62-0.77) for the TZ (Figs. 4 and 5). The model correctly classified $92 \%$ of $\mathrm{PZ}$ cases and $62 \%$ of $\mathrm{TZ}$ cases.

For areas that were scored by the radiologists as (highly) likely or unlikely (Likert $1 / 5,2 / 5,4 / 5$ and $5 / 5$ ) to contain significant cancer, the zone-specific LR models had similar performance with the radiologists (Table 4)

- Radiologist A (Likert 1/5, 2/5, 4/5 and 5/5): The diagnostic model had an ROC-AUC $=0.77$ (95\% CI 0.68-0.85) in the PZ and 0.66 (95\% CI $0.58-0.75)$ in the TZ. Radiologist A had an ROC-AUC $=0.80$ (95\% CI 0.72 0.87 ) in the PZ and 0.69 (95\% CI $0.60-0.78$ ) in the TZ.

- Radiologist B (Likert 1/5, 2/5, 4/5 and 5/5): The diagnostic model had an ROC-AUC $=0.73(95 \%$ CI $0.65-0.82)$ in the PZ and 0.65 (95\% CI $0.57-0.74)$ in the TZ. Radiologist B had an ROC-AUC $=0.74$ (95\% CI 0.65 0.83 ) in the PZ and 0.73 (95\% CI $0.66-0.81$ ) in the TZ.

\section{Discussion}

There were four principal observations. Firstly, we confirm that the performance of both PZ and TZ LR models for classification of patients with significant tumour was comparable to experienced radiologists.

Secondly, we highlight that zone-specific mp-MRI LR models may help classify equivocal radiologist-scored areas (Likert 3/5).

Thirdly, the examined zone-specific mp-MRI LR models reliably classified significant cancers both in the PZ and the $\mathrm{TZ}$ (the percentage of patients classified as false positives ranged from 2 to $10 \%$ ). In agreement with the PI-RADS v2 guidelines [21], the contribution of ADC is higher than the contribution of T2 in the PZ model, whereas for the TZ model, T2 has higher contribution than ADC.

Fourthly, by applying $1.5 \mathrm{~T}$ mp-MRI-derived LR models to an independent $3 \mathrm{~T}$ mp-MRI dataset, we have confirmed the external validity and generalizability of the previously published models [5, 6]. The generalizability can be attributed to the selected quantitative MR parameters, which are calculated as relative signal changes hence are less affected by the differences during data acquisition between the training (1.5 T magnet) and the independent (3 T magnet) cohort. This finding is also supported by a previous study [6] where the examined TZ diagnostic model was applied on a temporal cohort of $\mathrm{TZ}$ prostate cancer patients imaged at the same $1.5 \mathrm{~T}$ scanner. This study showed similar results of the temporal validation (ROC-AUC $=0.67$ ) with the independent validation illustrated here $(\mathrm{ROC}-\mathrm{AUC}=0.68)$.

Our study evaluated previously reported $1.5 \mathrm{~T}$ mp-MRI zone-specific diagnostic model performance on an 
Table 3 Performance of $\mathrm{PZ}$ and $\mathrm{TZ}$ models applied to $3 \mathrm{~T} \mathrm{mp-}$ MRI PICTURE trial dataset at (i) Youdens index derived probability threshold and (ii) at set 50\% specificity derived probability threshold

\begin{tabular}{lllll}
\hline \multicolumn{4}{l}{ Youdens index threshold } & \multicolumn{2}{l}{$50 \%$ specificity threshold } \\
& Specificity & Sensitivity & Specificity & Sensitivity \\
PZ model & 0.57 & 0.84 & 0.51 & 0.90 \\
TZ model & 0.32 & 0.84 & 0.23 & 0.90 \\
Sub-analysis & on equivocal group (Likert score 3) & & \\
& Radiologist A & Radiologist B & Radiologist A & Radiologist B \\
& Specificity/sensitivity & Specificity/sensitivity & Specificity/sensitivity & Specificity/sensitivity \\
PZ model & $0.42 / 0.75$ & $0.52 / 0.61$ & $0.39 / 0.90$ & $0.48 / 0.78$ \\
TZ model & $0.36 / 0.89$ & $0.50 / 0.73$ & $0.14 / 0.95$ & $0.26 / 0.91$ \\
\hline
\end{tabular}

independent cohort of patients scanned on a 3-T MR system to evaluate their ability to aid radiologists. Separate validation of the models was performed for areas identified as equivocal that may practically aid experienced radiologists in predicting tumour. Previous work from our group [22] also reported the reverse scenario where zone-specific LR models were trained in the $3 \mathrm{~T}$ mp-MRI dataset and applied on the $1.5 \mathrm{~T} \mathrm{mp-MRI}$ dataset. The reported ROC-AUCs were 0.76 (\%CI 0.68-0.97) in the PZ and 0.77 (\%CI 0.66-0.89) in the TZ. Following a sub-analysis, for the cases that were scored as indeterminate, the ROC-AUCs were 0.72 (\%CI 0.50-0.94) in the PZ and 0.77 (\%CI $0.55-0.96)$ in the TZ. The reported ROC-AUCs are similar to the ones reported in this work especially in the $\mathrm{PZ}$, but even in the TZ, the ROC-AUC differences are not significantly different $(p>0.05)$.

In the current study, even though expert radiologists reported the mp-MRI, diagnostic accuracy remained modest overall, and almost a quarter of regions evaluated were classified as equivocal for clinically significant cancer. This is in accordance with other studies where up to $40 \%$ of the cases were reported as indeterminate (using either the PI-RADS or the
Likert scoring) for the presence of cancer by the radiologist after reviewing mp-MRI dataset [23]. Approximately $40 \%$ of the cases classified as equivocal, contained significant cancer on TPM histology in the PZ, and $46 \%$ of these contained significant cancer on TPM histology in the TZ.

We found similar ROC-AUCs for the radiologist consensus score and the derived mp-MRI diagnostic models. At first glance, this suggests that the models would not benefit experienced radiologists working in consensus. However, the ROC-AUC for radiologists' evaluation does not reflect the uncertainty of equivocal scores. When the diagnostic models were evaluated on the radiologist A equivocal ROIs (51 in the PZ and 41 in the TZ), 86\% of PZ model predicted cases and $64 \%$ of $\mathrm{TZ}$ model predicted cases were correctly identified. Similarly, when the diagnostic models were evaluated on the radiologist $\mathrm{B}$ equivocal ROIs, 92\% of PZ model predicted cases and $62 \%$ of $\mathrm{TZ}$ model predicted cases were correctly identified. Following a sub-analysis on the cases scored as indeterminate by the radiologists the percentages of false positives predicted by the diagnostic models ranged from 2 to $10 \%$. These results indicate that the zone-specific diagnostic
Fig. 4 ROC curve for LR model classification of significant cancer for equivocal radiologist scored (Likert 3/5) PZ lesions from radiologist $\mathrm{A}, \mathrm{ROC}-\mathrm{AUC}=0.65$ (95\% CI $0.50-0.80)$, and radiologist $\mathrm{B}, \mathrm{ROC}-\mathrm{AUC}=0.67$ (95\% CI 0.51-0.82)

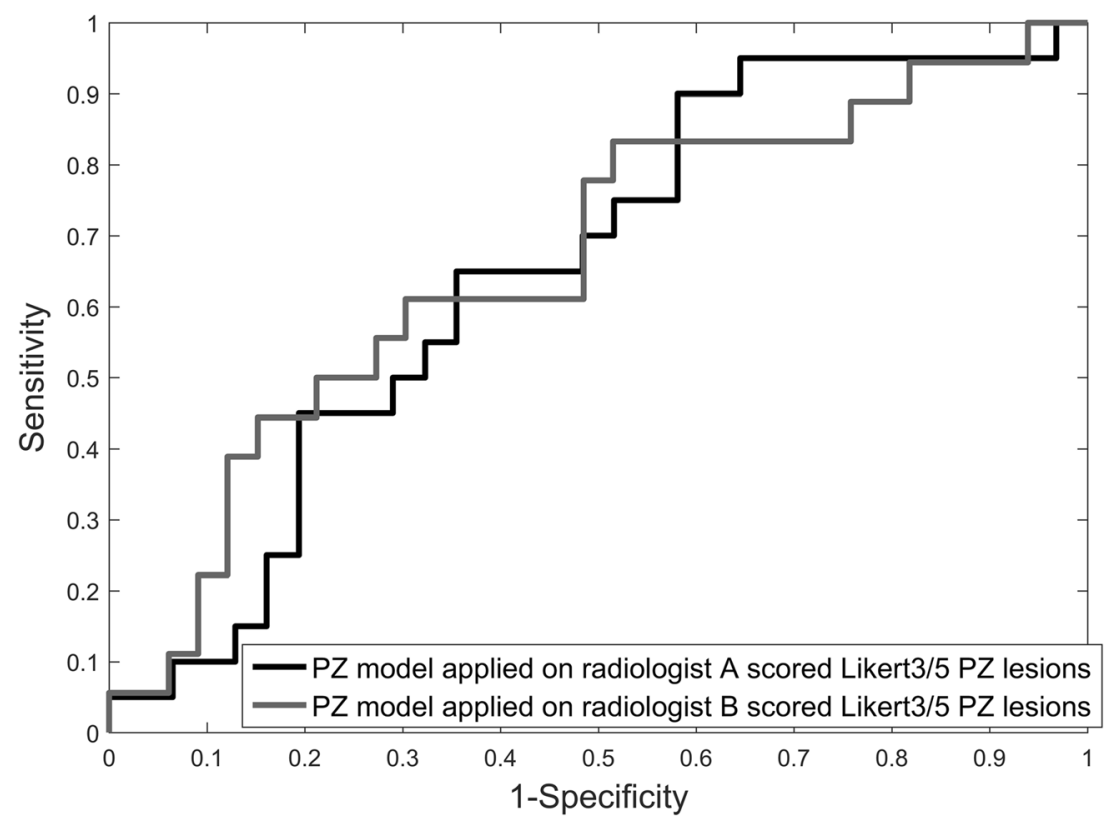


Fig. 5 ROC curve for LR model classification of significant cancer for equivocal radiologist scored (Likert 3/5) TZ lesions from radiologist $\mathrm{A}, \mathrm{ROC}-\mathrm{AUC}=0.74$ (95\% CI 0.58-0.90), and radiologist $\mathrm{B}, \mathrm{ROC}-\mathrm{AUC}=0.69$ (95\% CI 0.62-0.77)

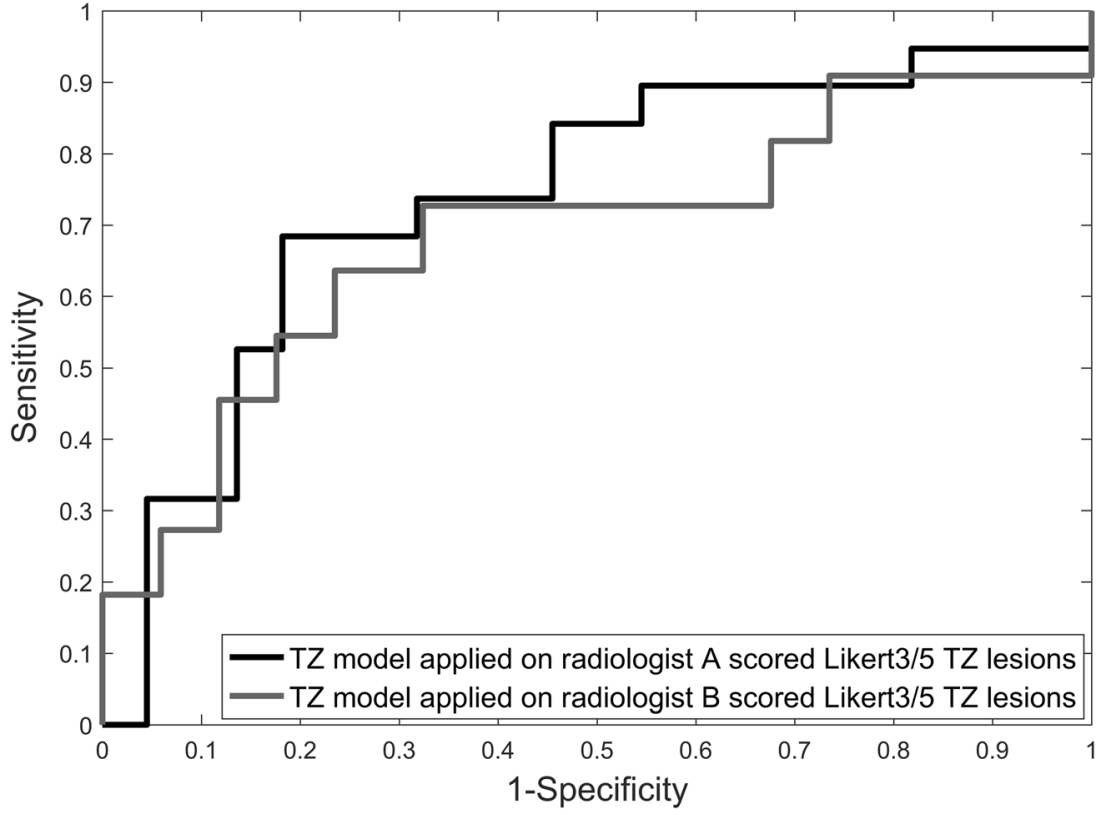

models are trustworthy when classifying an indeterminate case as significant cancer and that the diagnostic model may indeed have the potential to aid even experienced radiologists.

Evidence is mounting for the use of mp-MRI to triage patients prior to performing a prostate biopsy. Negative mpMRI is associated with about 1-2 in 20 risks of clinically significant disease [24], whereas positive mp-MRI is associated with about 3 in 10 risks of clinically insignificant disease $[25,26]$. Equivocal mp-MRI scores would tend to be biopsied. A tool to assist radiologists to avoid an equivocal category and correctly identify those patients with a low risk of clinically significant tumour would be of considerable value. Using the clinically relevant probability threshold on the indeterminate cases, the examined zone-specific mp-MRI models had (i) sensitivity $90 \%$ in the $\mathrm{PZ}$ and $95 \%$ in the $\mathrm{TZ}$ for radiologist $\mathrm{A}$ and (ii) sensitivity $78 \%$ in the $\mathrm{PZ}$ and $91 \%$ in the $\mathrm{TZ}$ for radiologist $\mathrm{B}$.

Our study has several limitations. We were reliant upon visual matching of the Barzell zone histology on TPM and ROIs on mp-MRI. Therefore, the results may be influenced by misregistration errors. Although no biopsy is free from sampling error [27], we used TPM to address as much of the systematic error inherent to TRUS biopsy as possible [13, 28]. TPM also enables sampling in the anterior gland. Radical prostatectomy specimens could not act as an alternative for this study, as they would suffer from a large amount of
Table 4 ROC AUC of PZ and TZ LR models and comparative experienced radiologist performance for areas that were scored by the radiologists as (highly) likely or unlikely (Likert $1 / 5,2 / 5,4 / 5$ and $5 / 5$ ) to contain significant cancer

\begin{tabular}{|c|c|c|c|c|}
\hline & \multirow[t]{2}{*}{ Area } & \multirow[t]{2}{*}{ Std. error } & \multicolumn{2}{|c|}{ Asymptotic $95 \%$ confidence interval } \\
\hline & & & Lower bound & Upper bound \\
\hline \multicolumn{5}{|c|}{$\mathrm{PZ}$ areas scored by radiologist $\mathrm{A}$ as Likert $=1 / 5,2 / 5,4 / 5$ and $5 / 5$} \\
\hline PZ model & 0.77 & 0.04 & 0.68 & 0.85 \\
\hline Radiologist A & 0.80 & 0.03 & 0.72 & 0.87 \\
\hline \multicolumn{5}{|c|}{$\mathrm{PZ}$ areas scored by radiologist $\mathrm{B}$ as Likert $=1 / 5,2 / 5,4 / 5$ and $5 / 5$} \\
\hline PZ model & 0.73 & 0.04 & 0.65 & 0.82 \\
\hline Radiologist B & 0.74 & 0.04 & 0.65 & 0.83 \\
\hline \multicolumn{5}{|c|}{$\mathrm{TZ}$ areas scored by Radiologist $\mathrm{A}$ as Likert $=1 / 5,2 / 5,4 / 5$ and $5 / 5$} \\
\hline TZ model & 0.66 & 0.05 & 0.58 & 0.75 \\
\hline Radiologist A & 0.69 & 0.04 & 0.60 & 0.78 \\
\hline \multicolumn{5}{|c|}{$\mathrm{TZ}$ areas scored by Radiologist $\mathrm{B}$ as Likert $=1 / 5,2 / 5,4 / 5$ and $5 / 5$} \\
\hline TZ model & 0.65 & 0.05 & 0.57 & 0.74 \\
\hline Radiologist B & 0.73 & 0.04 & 0.66 & 0.81 \\
\hline
\end{tabular}


selection bias given that they exclude men with no cancer and represent a minority of all men diagnosed [29]. Moreover, they also present technical challenges of accounting for distortion and gland shrinkage during fixing and the tissue loss in preparing the whole mount which is not trivial [30]. The relative merits of each reference standard have been considered before, but we believe TPM to be preferable in this patient cohort as it includes patients negative for cancer but presenting an elevated PSA.

\section{Conclusions}

In summary, we have assessed the performance of zonespecific mp-MRI diagnostic models for classification of peripheral and transition zone signal, demonstrated their similar performance to an experienced radiologist consensus and highlighted their ability to classify equivocal areas.

Acknowledgments Francesco Giganti is funded by the University College London (UCL) Graduate Research Scholarship and the Brahm $\mathrm{PhD}$ scholarship in memory of Chris Adams.

Funding This work was undertaken at UCLH/ UCL, which received a proportion of the funding from the NIHR Biomedical Research Centres funding scheme of the UK Department of Health. The work of HSS and SP was supported by the KCL/UCL Comprehensive Cancer Imaging Centre. The work of HUA is supported by core funding from the NIHR (UK) Imperial Biomedical Research Centre.

\section{Compliance with ethical standards}

Guarantor The scientific guarantor of this publication is Shonit Punwani.

Conflict of interest The authors of this manuscript declare no relationships with any companies, whose products or services may be related to the subject matter of the article.

Statistics and biometry No complex statistical methods were necessary for this paper.

Informed consent Written informed consent was waived by the Institutional Review Board.

Ethical approval Institutional Review Board approval was obtained.

\author{
Methodology \\ - Retrospective \\ - Experimental \\ - Performed at one institution
}

Open Access This article is distributed under the terms of the Creative Commons Attribution 4.0 International License (http:// creativecommons.org/licenses/by/4.0/), which permits unrestricted use, distribution, and reproduction in any medium, provided you give appropriate credit to the original author(s) and the source, provide a link to the Creative Commons license, and indicate if changes were made.

\section{References}

1. National Prostate Cancer Audit Fourth Year Annual Report Results of the NPCA Prospective Audit in England and Wales for men diagnosed 1 April 2015-March 2016. Available via https:// www.npca.org.uk. Accessed 01 Aug 2018

2. Renard-Penna R, Mozer P, Cornud F et al (2015) Prostate imaging reporting and data system and Likert scoring system: multiparametric MR imaging validation study to screen patients for initial biopsy. Radiology 275:458-468

3. Brizmohun Appayya M, Sidhu HS, Dikaios N et al (2018) Characterizing indeterminate (Likert-score 3/5) peripheral zone prostate lesions with PSA density, PI-RADS scoring and qualitative descriptors on multiparametric MRI. Br J Radiol 91:20170645

4. Simmons LA, Ahmed HU, Moore CM et al (2014) The PICTURE study - prostate imaging (multi-parametric MRI and Prostate HistoScanning ${ }^{\mathrm{TM}}$ ) compared to transperineal ultrasound guided biopsy for significant prostate cancer risk evaluation. Contemp Clin Trials 37:69-83

5. Dikaios N, Alkalbani J, Abd-Alazeez M et al (2015) Zone-specific logistic regression models improve classification of prostate cancer on multi-parametric MRI. Eur Radiol 25:2727-2737

6. Dikaios N, Alkalbani J, Sidhu HS et al (2015) Logistic regression model for diagnosis of transition zone prostate cancer on multiparametric MRI. Eur Radiol 25:523-532

7. Le JD, Tan N, Shkolyar E et al (2015) Multifocality and prostate cancer detection by multiparametric magnetic resonance imaging: correlation with whole-mount histopathology. Eur Urol 67:569-576

8. de Rooij M, Hamoen EH, Fütterer JJ, Barentsz JO, Rovers MM (2014) Accuracy of multiparametric MRI for prostate cancer detection: a meta-analysis. AJR Am J Roentgenol 202:343-351

9. Wang S, Burtt K, Turkbey B, Choyke P, Summers RM (2014) Computer aided-diagnosis of prostate cancer on multiparametric MRI: a technical review of current research. Biomed Res Int 2014:789561

10. Ahmed HU, El-Shater Bosaily A, Brown LC et al (2017) Diagnostic accuracy of multi-parametric MRI and TRUS biopsy in prostate cancer (PROMIS): a paired validating confirmatory study. Lancet 389:815-822

11. Brizmohun Appayya M, Adshead J, Ahmed HU et al (2018) National Implementation of multi-parametric MRI for prostate cancer detection - recommendations from a UK consensus meeting. BJU Int 122:13-25

12. Crawford ED, Rove KO, Barqawi AB et al (2013) Clinicalpathologic correlation between transperineal mapping biopsies of the prostate and three-dimensional reconstruction of prostatectomy specimens. Prostate 73:778-787

13. Ahmed HU, Hu Y, Carter T et al (2011) Characterizing clinically significant prostate cancer using template prostate mapping biopsy. J Urol 186:458-464

14. Crawford ED, Wilson SS, Torkko KC et al (2005) Clinical staging of prostate cancer: a computer-simulated study of transperineal prostate biopsy. BJU Int 96:999-1004

15. Stark JR, Perner S, Stampfer MJ et al (2009) Gleason score and lethal prostate cancer: does $3+4=4+3$ ? J Clin Oncol 27:3459

16. Zelhof B, Lowry M, Rodrigues G, Kraus S, Turnbull L (2009) Description of magnetic resonance imaging-derived enhancement variables in pathologically confirmed prostate cancer and normal peripheral zone regions. BJU Int 104:621-627

17. Youden WJ (1950) Index for rating diagnostic tests. Cancer 3:3235

18. Perkins NJ, Schisterman EF (2005) The Youden index and optimal cut-point corrected for measurement error. Biom J 47:428-441 
19. Reiser B (2009) Measuring the effectiveness of diagnostic markers in the presence of measurement error through the use of ROC curves. Stat Med 19:2115-2129

20. Schisterman EF, Perkins NJ (2007) Confidence intervals for the Youden index and corresponding optimal cut-point. Commun Stat Simul Comput 36:549-563

21. Barentsz JO, Weinreb JC, Verma S et al (2016) Synopsis of the PIRADS v2 guidelines for multiparametric prostate magnetic resonance imaging and recommendations for use. Eur Urol 69:41-49

22. Dikaios N, Johnston EW, Sidhu HS et al (2017) Deep learning to improve prostate cancer diagnosis. Joint Annual Meeting ISMRMESMRMB, Honolulu, USA. Available via http://cds.ismrm.org/ protected/17MPresentations/abstracts/0669.html. Accessed 03 Aug 2018

23. Liddell H, Jyoti R, Haxhimolla HZ (2015) mp-MRI prostate characterised PIRADS 3 lesions are associated with a low risk of clinically significant prostate cancer - a retrospective review of 92 biopsied PIRADS 3 lesions. Curr Urol 8:96-100

24. Puech P, Sufana Iancu A, Renard B, Villers A, Lemaitre L (2012) Detecting prostate cancer with MRI - why and how. Diagn Interv Imaging 93:268-278
25. Moore CM, Robertson NL, Arsanious N et al (2013) Image-guided prostate biopsy using magnetic resonance imaging-derived targets: a systematic review. Eur Urol 63:125-140

26. Haffner J, Lemaitre L, Puech P et al (2011) Role of magnetic resonance imaging before initial biopsy: comparison of magnetic resonance imaging-targeted and systematic biopsy for significant prostate cancer detection. BJU Int 108:171178

27. Ahmed HU, Emberton M, Kepner G, Kepner J (2012) A biomedical engineering approach to mitigate the errors of prostate biopsy. Nat Rev Urol 9:227-23113

28. Hoeks CM, Barentsz JO, Hambrock T et al (2011) Prostate cancer: multiparametricMR imaging for detection, localization, and staging. Radiology 261:46-66

29. Roy C, Foudi F, Charton J et al (2013) Comparative sensitivities of functional MRI sequences in detection of local recurrence of prostate carcinoma after radical prostatectomy or external-beam radiotherapy. AJR Am J Roentgenol 200:361-368

30. Turkbey B, Pinto PA, Mani H et al (2010) Prostate cancer: value of multiparametric MR imaging at $3 \mathrm{~T}$ for detection - histopathologic correlation. Radiology 255:89-99 